\title{
Incidentes e eventos adversos em segurança do paciente: uma série temporal de 2019 a 2020
}

\section{Incidents and adverse events in patient safety: a time series from 2019 to 2020}

Tathiane Ribeiro da Silva1', Douglas Eulálio Antunes²

1. ORCID: https://orcid.org/0000-0003-2898-8439 Especialista em Auditoria e Pesquisa. Empresa Brasileira de Serviços Hospitalares (EBSERH), Uberaba, Minas Gerais, Brasil. E-mail: tathianeauditora@gmail.com

2. ORCID: https://orcid.org/0000-0001-8091-7158 Doutor em Ciências da Saúde. Universidade Federal de Uberlândia, Minas Gerais, Brasil. E-mail: douglas.eulalio1@ gmail.com

CONTATO: Autor correspondente: Douglas Eulálio Antunes | Endereço: Av. Pará, Bloco 2u, 1720 - Umuarama, Uberlândia - MG, Brasil Telefone: (34) 99111-8446 E-mail: douglas.eulalio1@gmail.com

RESUMO Trata-se de uma análise de série temporal que objetivou caracterizar o perfil de incidentes e eventos adversos associados à segurança do paciente, na região sudeste entre julho de 2019 e junho de 2020. Para fins de associação e análise de tendência, foram utilizados os testes $G$ de independência e Mann-Kendall, respectivamente. Do total de 59.822 incidentes, $76,9 \%$ (46.336) acarretaram eventos adversos ao paciente. Houve associação entre falhas envolvendo cateteres venosos e dano leve nos estados do Espírito Santo $(87,4 \% ; p<0,0001)$ e Rio de Janeiro leve $(66,2 \% ; p<0,0001)$. Em São Paulo (24,1\%; $p<0,0001)$ e Minas Gerais (30\%; $p<0,0001)$, as lesões por pressão associaram-se ao dano moderado. Quanto ao número de notificação de incidentes, registraram-se tendências decrescentes no Espirito Santo e Rio de Janeiro. Portanto, evidenciou-se elevado número de eventos adversos de dano moderado, além de tendências decrescentes nas notificações em alguns estados requerendo monitoramento e avaliação contínua de falhas na assistência para evitar subnotificações. 
DESCRITORES: Segurança do Paciente. Gestão de Segurança. Estudos de Séries Temporais.

ABSTRACT This is a time series analysis that aimed to characterize the profile of incidents and adverse events associated with patient safety in the Southeast region between July 2019 and June 2020. For association and trend analysis purposes, the $\mathrm{G}$ independence and Mann-Kendall tests were used, respectively. Of a total of 59,822 incidents, $76.9 \%(46,336)$ caused adverse events to the patient. There was an association between failures involving venous catheters and mild damage in the states of Espírito Santo $(87.4 \% ; p<0.0001)$ and mild Rio de Janeiro $(66.2 \% ; p<0.0001)$. In São Paulo $(24.1 \%$; $<0.0001)$ and Minas Gerais $(30 \% ; p<0.0001)$, pressure injuries were associated with moderate damage As for the number of incident notifications, decreasing trends were registered in Espirito Santo and Rio de Janeiro. Therefore, there was a high number of adverse events of moderate harm, in addition to decreasing trends in notifications in some states, requiring continuous monitoring and evaluation of care failures to avoid underreporting.

DESCRIPTORS: Patient Safety. Sacurity Management. Time Series Studies. 


\section{INTRODUÇÃO}

Segundo a Organização Mundial de Saúde (OMS), segurança do paciente consiste na redução, a um mínimo aceitável, do risco de danos desnecessários associados à saúde. Quando um evento atinge o paciente e não acarreta danos, trata-se de um incidente. Por outro lado, um incidente que causa danos ao paciente é caracterizado como um evento adverso ${ }^{1}$.

O movimento que corroborou para o surgimento da segurança do paciente iniciou na década de $90 \mathrm{com}$ a publicação de estudo conduzido pelo Instituto de Medicina que estimou em torno de 44.000 a 98.000 mortes anuais nos Estados Unidos associadas a danos evitáveis em hospitais ${ }^{2}$.

No Brasil, o foco na assistência isenta de danos ganhou notoriedade na década de 90 quando as instituições públicas e privadas almejaram a criação de estratégias com a finalidade de garantir assistência de qualidade aos pacientes ${ }^{3}$.

No ano de 2002 na Assembleia Mundial em Saúde foram discutidos pela primeira vez a qualidade do cuidado e segurança do paciente. Já em 2004 a Organização Mundial da Saúde (OMS) criou a World Alliance for Patient Safety com o objetivo de reduzir o problema do cuidado inseguro, propondo "Soluções para a Segurança do Paciente". E, em parceria com a Joint Commission International (JCl), foram criadas como soluções as seis Metas Internacionais de Segurança do Paciente com o objetivo de promover meIhorias específicas em áreas problemáticas na assistência ${ }^{4}$.

Mesmo com a implementação das seis Metas internacionais de Segurança do Paciente, atualmente estabelecidas 7 metas, a incidência estimada para os eventos adversos tem se mantido elevada em instituições hospitalares com variação entre 5,4 a $14,2 \%$ segundo levantamento de 2004 a 2019 em uma revisão integrativa da literatura ${ }^{5}$. Além disso, de acordo com a Organização Mundial de saúde, existe uma carga econômica associada a erros de medicação que acarretou um custo global de 42 bilhões de dólares anuais $^{6}$. Outro dado importante está relacionado à cirurgia insegura, visto que, anualmente foram quase 7 milhões de pacientes acometidos por complicações com 1 milhão deles indo a óbito durante ou imediatamente após cirurgia ${ }^{7}$.

Assim, pautada na potencial mitigação dos eventos adversos, é de extrema relevância a gestão dos riscos e investigação destes a fim de prevenir danos aos pacientes. A Agência Nacional de Segurança do Paciente, (National Patient Safety Agency - NPSA) do Sistema Nacional de Saúde (National Health Service - NHS) do Reino Unido, define gestão de risco como o processo de identificação, análise, e gerenciamento dos riscos potenciais por meio do conhecimento das circunstâncias (perigos), frequência e consequências (gravidade) dos danos ${ }^{8}$. Além disso, a NPSA propõe a implementação de 7 
passos para a segurança do paciente, como: promoção de uma cultura de segurança; liderar e apoiar a equipe, integração das atividades de gestão dos riscos; promover notificação de incidentes; estímulo da participação do paciente; compartilhamento de aprendizado sobre segurança e implementar práticas de segurança ${ }^{8-9}$.

Objetivou-se caracterizar o perfil das notificações de incidentes e eventos adversos relacionados à segurança do paciente ocorridos entre julho de 2019 a junho de 2020 na região sudeste, a fim de sumarizar os principais achados; descrever associações entre falhas e tipos de dano; e evidenciar tendências a longo do tempo analisado.

\section{MÉTODO}

\section{Tipo de estudo e amostra}

Trata-se de uma análise de série temporal entre julho de 2019 a junho de 2020 utilizando-se dados de domínio público envolvendo registros relacionados às notificações de incidentes e eventos adversos referentes à segurança do paciente reportadas pelas instituições de saúde dos estados do Espírito Santo, Minas Gerais, Rio de Janeiro e São Paulo ao Sistema Nacional de Notificações para a Vigilância Sanitária - NOTIVISA ${ }^{10}$.

\section{Dados secundários}

As principais variáveis referentes à segurança do paciente foram: faixa etária dos pacientes acometidos; frequência mensal de incidentes e eventos adversos de segurança do paciente; tipo de incidente e eventos adversos e o grau do dano associado a esses eventos.

De acordo com o NOTIVISA as falhas durante a assistência à saúde são compostas por um amplo rol de procedimentos envolvendo principalmente os processos de triagem, check-up, diagnóstico, meios complementares de diagnóstico/procedimento/tratamento/ intervenção, assistência geral e contenção física.

As informações levantadas nesse estudo referem-se a dados secundários de domínio público que pertencem ao NOTIVISA da Agência Nacional de Vigilância Sanitária (ANVI$\mathrm{SA}$ ). Todos os dados foram coletados entre o dia primeiro de setembro a 01 de outubro de 2020.

\section{Critérios de inclusão e exclusão}

O estudo incluiu dados sobre segurança do paciente dos estados do Sudeste do Brasil que constavam na plataforma NOTIVISA da Agência Nacional de Vigilância Sanitária 
entre os períodos de julho de 2019 a junho de 2020. Os dados secundários, anteriores ou posteriores ao referido período de coleta, não fizeram parte desta tabulação.

\section{Análise estatística}

$O$ teste $G$ de independência foi utilizado para verificar a associação entre valores absolutos dos diversos tipos de incidentes e eventos adversos e o grau do dano nas diferentes unidades federativas da região Sudeste brasileira.

Dados referentes ao número de notificações de incidentes e eventos adversos a cada mês e por faixa etária da série temporal foi avaliada quanto a presença de tendência, por meio do teste de Mann-Kendall e estacionariedade (teste Kwiatkowski-Phillips-Schmidt-Shin, KPSS).

A análise foi processada nos programas GraphPad Prism 4.04 (GraphPad Software, Inc., San Diego, CA) e Action Stat Pro 3.7 (ActionStat Software, Campinas, SP). As hipóteses nulas e alternativas foram testadas a um nível de significância a de $5 \%(0,05)$.

\section{Considerações éticas}

Este estudo dispensa a apreciação do Comitê de Ética em Pesquisa com Seres Humanos devido aos dados serem apresentados em bases de dados de domínio público e divulgados na plataforma da Agência Nacional de Vigilância Sanitária (NOTIVISA).

\section{RESULTADOS}

\section{Caracterização dos eventos adversos entre unidades federativas}

Na região sudeste, do total de 59.822 incidentes, 76,9\% (46.336) foram caracterizados como eventos adversos. Nota-se que 79,5\% (36.848/46.336) dos incidentes foram caracterizados, como grau do dano leve. Os incidentes moderados, graves e aqueles que ocasionaram óbitos representaram 16,7\% (7.768/46.336); 2,8\% (1.316/46.336) e $0,8 \%(404 / 46.336)$ respectivamente.

Os Estados de Minas Gerais e São Paulo notificaram um total de 30.477 e 21.449 incidentes que representaram, respectivamente, 50,9\% e 35,9\% do total de registros da região sudeste. Já o Espírito Santo e Rio de Janeiro notificaram apenas 7\% (4.208) e $6,2 \%$ (3.688) do total de incidentes da região sudeste.

$\mathrm{Na}$ análise segundo o tipo de incidente e evento adverso, houve predomínio daqueles associados a falhas durante a assistência à saúde (26,5\%;15.831/59.822); envolvendo cateteres venosos $(19 \% ; 11377 / 59.822)$ e lesões por pressão $(17,7 \% ; 10.602 / 59.822)$. Na 
avaliação dos incidentes e eventos adversos que imperaram por estado, no Espírito Santo $43 \%$ (1.809/4.208) foram relacionados a falhas envolvendo cateteres venosos e no Rio de Janeiro $30,9 \%(1.141 / 3.688)$ dos eventos também estiveram associados a esse mesmo incidente. Em Minas Gerais, as falhas durante assistência à saúde prevaleceram com 36,4\% (11.100/30.477) do total para o estado, enquanto que em São Paulo, as lesões por pressão representaram $21,6 \%$ (4.640/21.449) dos incidentes e eventos adversos.

As Tabelas 1 (primeiro grupo de variáveis) e 2 (segundo grupo de variáveis) apresentam as frequências dos tipos de incidentes e/ou eventos adversos por unidade federativa associados aos graus dos danos. Destaca-se na Tabela 1, a associação entre as falhas envolvendo cateteres venosos e o grau de dano leve nos estados do Espírito Santo $(87,4 \% ; 1.581 / 1.809 ; p<0,0001)$ e Minas Gerais $(88,5 \% ; 3.972 / 4.490 ; p<0,0001)$. Ainda na Tabela 1, no estado de Minas Gerais, houve relação entre lesões por pressão e o dano moderado $(30 \% ; 1.508 / 5.033 ; p<0,0001)$. No Rio de Janeiro, a queda do paciente associou-se a nenhum dano $(58,7 \% ; 442 / 753 ; p<0,0001)$ e as falhas envolvendo cateteres esteve relacionada ao dano leve $(66,2 \% ; 755 / 1141 ; p<0,0001)$ (Tabela 1).

Em relação ao estado de São Paulo, as lesões por pressão estiveram associadas aos graus moderados $(24,1 \% ; 1.117 / 4.640 ; p<0,0001)$ enquanto que o incidente, queda do paciente, esteve relacionado a nenhum dano $(75,1 \% ; 1.566 / 3.708 ; p<0,0001)$.

As informações presentes na Tabela 2 apontaram associações entre notificações referentes às falhas durante a assistência à saúde e o grau de dano leve em todos os estados do Sudeste. Destaca-se que a maioria dos óbitos em todas as unidades federativas do Sudeste estiveram associadas também às falhas durante a assistência à saúde. 
Tabela 1. Tipos de Incidentes e Eventos Adversos (Primeiro grupo de variáveis) associados aos graus do dano por unidade federativa notificadora, série temporal entre julho de 2019 a junho de 2020

\begin{tabular}{|c|c|c|c|c|c|c|c|c|c|c|c|c|c|c|c|}
\hline \multirow{3}{*}{$\begin{array}{l}\text { Unidade Federati- } \\
\text { va notificadora }\end{array}$} & \multirow{3}{*}{$\begin{array}{l}\text { Grau do } \\
\text { Dano }\end{array}$} & \multicolumn{12}{|c|}{ Tipo de Incidente/ Evento adverso } & & \\
\hline & & \multicolumn{2}{|c|}{$\begin{array}{l}\text { Falha na identifica- } \\
\text { ção do paciente }\end{array}$} & \multicolumn{2}{|c|}{$\begin{array}{l}\text { Queda do } \\
\text { paciente }\end{array}$} & \multicolumn{2}{|c|}{$\begin{array}{l}\text { Lesão Por } \\
\text { pressão }\end{array}$} & \multicolumn{2}{|c|}{$\begin{array}{l}\text { Extubação Endo- } \\
\text { traqueal Acidental }\end{array}$} & \multicolumn{2}{|c|}{$\begin{array}{l}\text { Falhas envol- } \\
\text { vendo sondas }\end{array}$} & \multicolumn{2}{|c|}{$\begin{array}{l}\text { Falhas envolvendo } \\
\text { Cateter venoso }\end{array}$} & \multicolumn{2}{|c|}{ Teste G } \\
\hline & & $\mathbf{n}$ & $\%$ & $\mathbf{n}$ & $\%$ & $\mathbf{n}$ & $\%$ & $\mathbf{n}$ & $\%$ & $\mathbf{n}$ & $\%$ & $\mathbf{n}$ & $\%$ & G & $p$-valor \\
\hline \multirow{6}{*}{ Espírito Santo } & Nenhum & 138 & 95,2 & 117 & 44,2 & 12 & 3.0 & 0 & 0 & 171 & 40,8 & 112 & 6,2 & \multirow{6}{*}{1037,5} & \multirow{6}{*}{$<0,0001$} \\
\hline & Leve & 6 & 4,1 & 120 & 45,3 & 332 & 83,8 & 0 & 0 & 217 & 51,8 & 1581 & 87,4 & & \\
\hline & Moderado & 0 & 0 & 23 & 8,7 & 51 & 12,9 & 0 & 0 & 30 & 7,2 & 114 & 6,3 & & \\
\hline & Grave & 1 & 0,7 & 5 & 1,9 & 1.0 & 0,3 & 2 & 100 & 1 & 0,2 & 2 & 0,1 & & \\
\hline & Óbito & 0 & 0 & 0 & 0 & 0 & 0 & 0 & 0 & 0 & 0 & 0 & 0 & & \\
\hline & Total & 145 & 100 & 265 & 100 & 396 & 100 & 2 & 100 & 419 & 100 & 1809 & 100 & & \\
\hline \multirow{6}{*}{ Minas Gerais } & Nenhum & 705 & 74,4 & 809 & 31,3 & 82 & 1,6 & 0 & 0 & 366 & 19.7 & 178 & 4,0 & \multirow{6}{*}{5828,4} & \multirow{6}{*}{$<0,0001$} \\
\hline & Leve & 180 & 19,0 & 1478 & 57,2 & 3240 & 64,4 & 0 & 0 & 1328 & 71.3 & 3972 & 88,5 & & \\
\hline & Moderado & 58 & 6,1 & 256 & 9,9 & 1508 & 30,0 & 0 & 0 & 163 & 8,8 & 309 & 6,9 & & \\
\hline & Grave & 5 & 0,5 & 31 & 1,2 & 203 & 4 & 0 & 0 & 5.0 & 0,3 & 31 & 0,7 & & \\
\hline & Óbito & 0 & 0 & 12 & 0,5 & 0 & 0 & 0 & 0 & 0 & 0 & 0 & 0 & & \\
\hline & Total & 948 & 100 & 2586 & 100 & 5033 & 100 & 0 & 0 & 1862 & 100 & 4490 & 100 & & \\
\hline \multirow{6}{*}{ Rio de Janeiro } & Nenhum & 260 & 97 & 442 & 58,7 & 1 & 0.2 & 7 & 19,4 & 46 & 43,4 & 336 & 29,4 & \multirow{6}{*}{1330} & \multirow{6}{*}{$<0,0001$} \\
\hline & Leve & 7 & 2,6 & 271 & 36 & 352 & 66 & 15 & 41,7 & 51 & 48,1 & 755 & 66,2 & & \\
\hline & Moderado & 1 & 0,4 & 33 & 4,4 & 170 & 31,9 & 9 & 25 & 7 & 6,6 & 39 & 3,4 & & \\
\hline & Grave & 0 & 0 & 4 & 0,5 & 10 & 1,9 & 5 & 13,9 & 2 & 1,9 & 9 & 0,8 & & \\
\hline & Óbito & 0 & 0 & 3 & 0,4 & 0 & 0 & 0 & 0 & 0 & 0 & 2 & 0,2 & & \\
\hline & Total & 268 & 100 & 753 & 100 & 533 & 100 & 36 & 100 & 106 & 100 & 1141 & 100 & & \\
\hline \multirow{6}{*}{ São Paulo } & Nenhum & 326 & 75,1 & 1566 & 42,2 & 84 & 1,8 & 95.0 & 31,5 & 678 & 23,7 & 106 & 2,7 & \multirow{6}{*}{5045,7} & \multirow{6}{*}{$<0,0001$} \\
\hline & Leve & 93 & 21,4 & 1872 & 50,5 & 3277 & 70,6 & 138 & 45,7 & 2101 & 73,5 & 3389 & 86,1 & & \\
\hline & Moderado & 13 & 3 & 222 & 6,0 & 1117 & 24,1 & 57 & 18,9 & 76 & 2,7 & 420 & 10,7 & & \\
\hline & Grave & 2 & 0,5 & 43 & 1,2 & 162 & 3,5 & 7 & 2,3 & 3 & 0,1 & 19 & 0,5 & & \\
\hline & Óbito & 0 & 0 & 5 & 0,1 & 0 & 0 & 5 & 1,7 & 0 & 0 & 1 & 0 & & \\
\hline & Total & 434 & 100 & 3708 & 100 & 4640 & 100 & 302 & 100 & 2858 & 100 & 3935 & 100 & & \\
\hline
\end{tabular}

Fonte: Elaborado pelos autores (2021) 
Tabela 2. Tipos de Incidentes e Evento Adversos (Segundo grupo de variáveis) associados aos graus do dano por unidade federativa notificadora, série temporal entre julho de 2019 a junho de 2020

\begin{tabular}{|c|c|c|c|c|c|c|c|c|c|c|c|c|c|c|c|}
\hline \multirow{3}{*}{$\begin{array}{l}\text { Unidade Federa- } \\
\text { tiva notificadora }\end{array}$} & \multirow{3}{*}{$\begin{array}{c}\text { Grau do } \\
\text { Dano }\end{array}$} & \multicolumn{8}{|c|}{ Tipo de incidente/ Evento adverso } & & & & & & \\
\hline & & \multicolumn{2}{|c|}{$\begin{array}{l}\text { Falhas durante a as- } \\
\text { sistência à saúde }\end{array}$} & \multicolumn{2}{|c|}{$\begin{array}{l}\text { Falhas na adminis- } \\
\text { tração de dietas }\end{array}$} & \multicolumn{2}{|c|}{$\begin{array}{l}\text { Acidente do } \\
\text { Paciente }\end{array}$} & \multicolumn{2}{|c|}{$\begin{array}{l}\text { Evasão do } \\
\text { Paciente }\end{array}$} & \multicolumn{2}{|c|}{$\begin{array}{l}\text { Falhas nas ativida- } \\
\text { des administrativas }\end{array}$} & \multicolumn{2}{|c|}{$\begin{array}{l}\text { Falha na do- } \\
\text { cumentação }\end{array}$} & \multicolumn{2}{|c|}{ Teste G } \\
\hline & & $\mathbf{n}$ & $\%$ & $\mathbf{n}$ & $\%$ & $\mathbf{n}$ & $\%$ & $\mathrm{n}$ & $\%$ & $\mathbf{n}$ & $\%$ & $\mathbf{n}$ & $\%$ & $G$ & $p$-valor \\
\hline \multirow{6}{*}{ Espírito Santo } & Nenhum & 165 & 18,1 & 37 & 80,4 & 0 & 0,0 & 0 & 0,0 & 0 & 0,0 & 0 & 0,0 & \multirow{6}{*}{93,1} & \multirow{6}{*}{$<0,0001$} \\
\hline & Leve & 721 & 78,9 & 9 & 19,6 & 0 & 0,0 & 0 & 0,0 & 24 & 96,0 & 0 & 0,0 & & \\
\hline & Moderado & 12 & 1,3 & 0 & 0,0 & 0 & 0,0 & 0 & 0,0 & 1 & 4,0 & 0 & 0,0 & & \\
\hline & Grave & 7 & 0,8 & 0 & 0,0 & 0 & 0,0 & 0 & 0,0 & 0 & 0,0 & 0 & 0,0 & & \\
\hline & Óbito & 9 & 1,0 & 0 & 0,0 & 0 & 0,0 & 0 & 0,0 & 0 & 0,0 & 0 & 0,0 & & \\
\hline & Total & 914 & 100 & 46 & 100 & 0 & 0,0 & 0 & 0,0 & 25 & 100 & 0 & 0,0 & & \\
\hline \multirow{6}{*}{ Minas Gerais } & Nenhum & 2616 & 23,6 & 192 & 23,8 & 35 & 5,6 & 539 & 83,4 & 0 & 0,0 & 637 & 79,0 & \multirow{6}{*}{1196,3} & \multirow{6}{*}{$<0,0001$} \\
\hline & Leve & 6135 & 55,3 & 558 & 69,2 & 495 & 78,9 & 68 & 10,5 & 0 & 0,0 & 151 & 18,7 & & \\
\hline & Moderado & 1858 & 16,7 & 46 & 5,7 & 78 & 12,4 & 24 & 3,7 & 0 & 0,0 & 15 & 1,9 & & \\
\hline & Grave & 355 & 3,2 & 8 & 1,0 & 10 & 1,6 & 15 & 2,3 & 0 & 0,0 & 3 & 0,4 & & \\
\hline & Óbito & 136 & 1,2 & 2 & 0,2 & 9 & 1,4 & 0 & 0,0 & 0 & 0,0 & 0 & 0,0 & & \\
\hline & Total & 11100 & 100 & 806 & 10 & 627 & 1000 & 646 & 100 & 0 & 0,0 & 806 & 100,0 & & \\
\hline \multirow{6}{*}{ Rio de Janeiro } & Nenhum & 139 & 27,5 & 0 & 0,0 & 0 & 0,0 & 63 & 80,8 & 27 & 81,8 & 100 & 90,9 & \multirow{6}{*}{580,3} & \multirow{6}{*}{$<0,0001$} \\
\hline & Leve & 247 & 48,9 & 0 & 0,0 & 0 & 0,0 & 8 & 10,3 & 6 & 18,2 & 4 & 3,6 & & \\
\hline & Moderado & 76 & 15,0 & 0 & 0,0 & 0 & 0,0 & 5 & 6,4 & 0 & 0,0 & 6 & 5,5 & & \\
\hline & Grave & 18 & 3,6 & 0 & 0,0 & 0 & 0,0 & 2 & 2,6 & 0 & 0,0 & 0 & 0,0 & & \\
\hline & Óbito & 25 & 5,0 & 0 & 0,0 & 0 & 0,0 & 0 & 0,0 & 0 & 0,0 & 0 & 0,0 & & \\
\hline & Total & 505 & 100 & 0 & 0,0 & 0 & 0,0 & 78 & 100 & 33 & 100 & 110 & 100 & & \\
\hline \multirow{6}{*}{ São Paulo } & Nenhum & 877 & 26,5 & 0 & 0,0 & 80 & 12,8 & 0 & 0,0 & 217 & 75,9 & 0 & 0,0 & \multirow{6}{*}{627,3} & \multirow{6}{*}{$<0,0001$} \\
\hline & Leve & 1861 & 56,2 & 0 & 0,0 & 459 & 73,3 & 0 & 0,0 & 60 & 21,0 & 0 & 0,0 & & \\
\hline & Moderado & 379 & 11,4 & 0 & 0,0 & 70 & 11,2 & 0 & 0,0 & 8 & 2,8 & 0 & 0,0 & & \\
\hline & Grave & 108 & 3,3 & 0 & 0,0 & 14 & 2,2 & 0 & 0,0 & 0 & 0,0 & 0 & 0,0 & & \\
\hline & Óbito & 87 & 2,6 & 0 & 0,0 & 3 & 0,5 & 0 & 0,0 & 1 & 0,3 & 0 & 0,0 & & \\
\hline & Total & 3312 & 100 & 0 & 0,0 & 626 & 100 & 0 & 0,0 & 286 & 100 & 0 & 0,0 & & \\
\hline
\end{tabular}

Fonte: Elaborado pelos autores (2021) 
Em relação ao número de notificações de incidentes, observou-se uma tendência decrescente nos estados do Espírito Santo (Mann-Kendall=-0.54; $p=0,0163$ ) e Rio de Janeiro (Mann-Kendall=-0.45; $p=0,0467$ ) ao longo da série temporal descrita entre 2019 e 2020 (Figura 1). Notou-se, portanto, ausência de estacionariedade, no Espírito Santo $(K P S S=0.51 ; p=0,0376)$ e Rio de janeiro (KPSS $=0.57 ; p=0,0250)$ (Figura 1).

Por outro lado, os estados de Minas Gerais (Mann-Kendall=-0.24; $p=0,3036$ ) e São Paulo (Mann-Kendall=0.12; $p=0,6312$ ) não apresentaram tendência quanto ao número de notificações ao longo da série temporal. Desse modo, foi registrada presença de estacionariedade no registro de notificações de incidentes e/ou eventos adversos nos serviços de saúde de Minas Gerais (KPSS=0.29; $p=0,1000$ ) e São Paulo (KPSS=0.12; $p=0,1000$ ) (Figura 1).

Figura 1. Número de notificações de incidentes e eventos adversos por unidade federativa da região sudeste entre os meses de julho de 2019 a junho de 2020

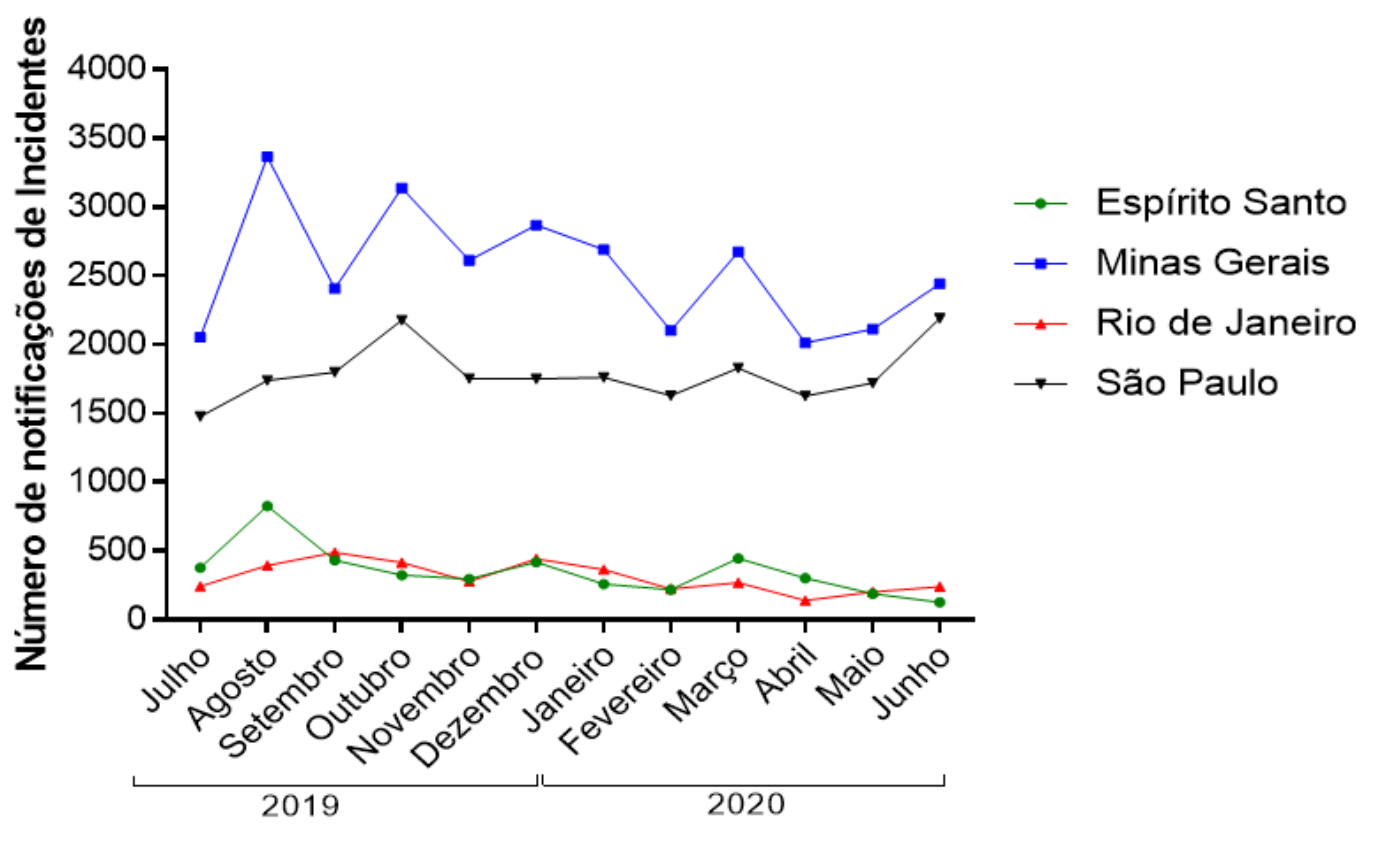

Fonte: Elaborado pelos autores (2021)

Quanto ao número de notificações de incidentes e lou eventos adversos por faixa etária, foram registradas tendências crescentes à medida que ocorreu aumento nas faixas etárias nos estados do Espírito Santo (Mann-Kendall=0.71; $p=0,0007$ ), Rio de Janeiro (Mann-Kendall=0.66; $p=0,0018$ ), Minas Gerais (Mann-Kendall $=0.64 ; p=0,0027$ ) e São Paulo (Mann-Kendall=0.58; $p=0,0060$ ) (Figura 2).

Ainda em relação ao número de notificações de incidentes e/ou eventos adversos por faixa etária, observa-se presença de estacionariedade entre as faixas etárias de 2 a 4 e de 12 a 17 anos em Minas Gerais, com média de 378,3 notificações e em São Paulo com 
média de 235 incidentes e/ou eventos adversos no mesmo período citado (Figura 2). Ressalta-se a tendência de declínio a partir da faixa etária entre 66 e 75 anos em Minas Gerais, com média de 4.190 incidentes e/ou eventos adversos; seguida por São Paulo, que registrou média de 2.911, mesmo predominando a tendência crescente no total de todas as faixas etárias, como relatado anteriormente (Figura 2).

Figura 2. Número de notificações de incidentes e eventos adversos por faixa etária nas 4 unidades federativas da região sudeste entre os meses de julho de 2019 a junho de 2020

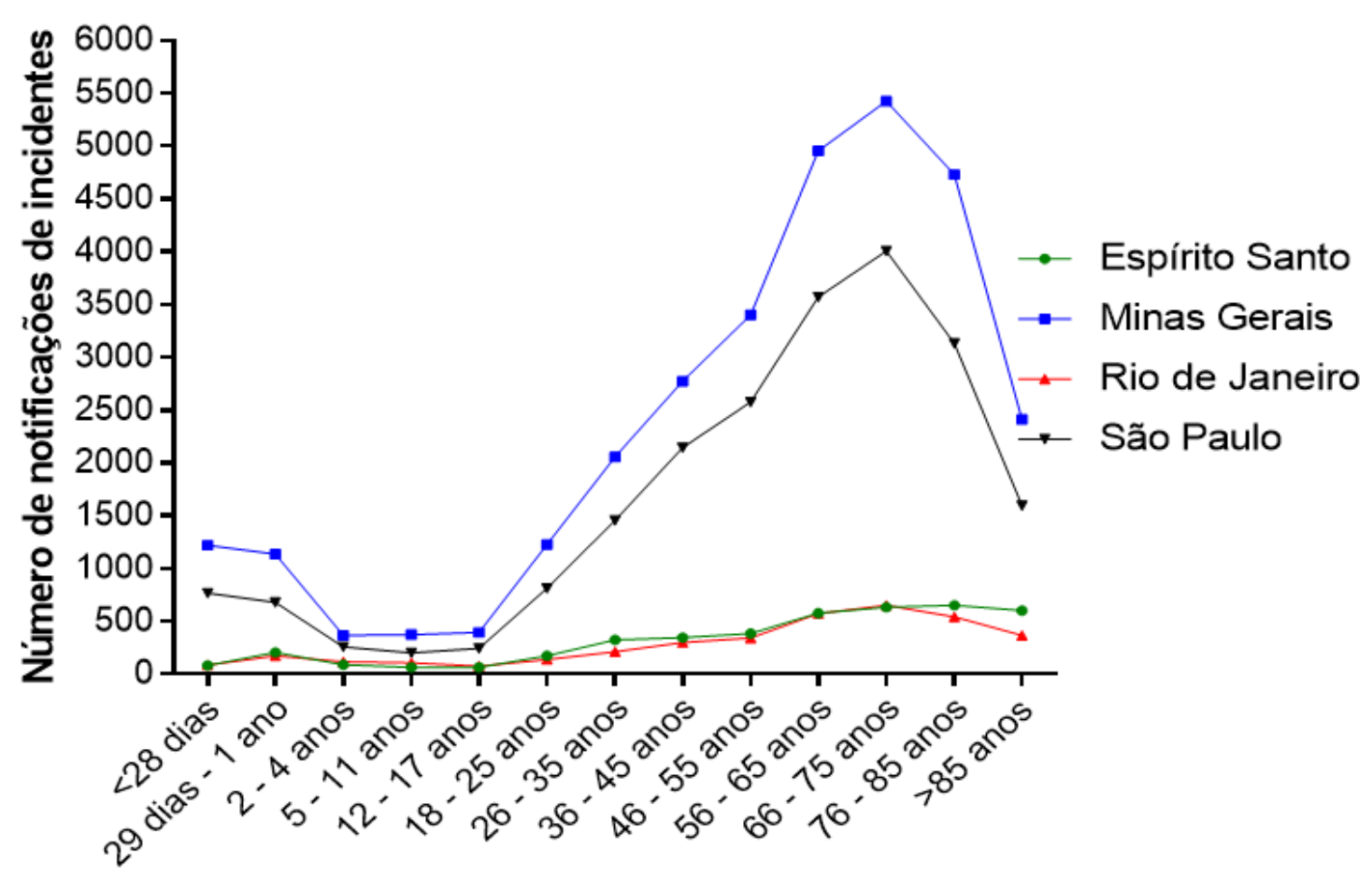

Fonte: Elaborado pelos autores (2021)

\section{DISCUSSÃO}

Aqueles incidentes e eventos adversos relacionados as falhas durante a assistência predominaram na região sudeste, sendo o mais notificado no estado de Minas Gerais na análise de série temporal entre julho de 2019 a junho de 2020. No entanto, ressalta-se que esse resultado pode estar conectado ao elevado rol de procedimentos agrupados nessa categoria de eventos, tais como processos de triagem, diagnóstico, procedimentos, tratamentos, intervenções gerais e dentre outros ${ }^{11}$. O erro de diagnóstico, incluso dentre as falhas durante a assistência, pode ser reduzido por meio de estratégias como uso de algoritmos clínicos e ferramentas de tomada de decisão ${ }^{12}$.

Os Incidentes e eventos adversos vinculados a cateteres venosos foram o segundo mais prevalente no Sudeste e o mais frequente no Espírito Santo e Rio de Janeiro. Estudo observacional revelou elevada incidência de eventos adversos envolvendo cateteres 
venosos periféricos, tais como flebites, seguidas por infiltração e oclusão de lúmen ${ }^{13}$. Quanto as complicações associadas a cateteres venosos centrais, estudo apontou aquelas ocorridas durante a inserção do cateter como o hematoma, e principalmente, as infecções causadas por Staphylococcus epidermidis e Staphylococcus aureus em unidades de terapia intensiva ${ }^{14}$.

As lesões por pressão foram o terceiro evento mais prevalente no Sudeste e predominou em São Paulo. A prevalência de lesões por pressão variou, segundo alguns estudos, entre $3,3 \%$ e 14,9\% com mais da metade delas adquiridas no hospital, além de acarretar complicações como infecções, tempo de internação prolongado e gastos hospitalares exorbitantes ${ }^{15-16}$.

Um estudo prospectivo realizado na França mostrou que os eventos adversos relacionados ao uso de cateteres venosos periféricos estiveram associados à flebite, hematoma e escape de líquido o que corroborou com os achados referentes às falhas envolvendo cateteres venosos e sua associação com grau de dano leve ${ }^{17}$.

Embora um estudo prévio tenha citado o grau de dano moderado em apenas 10,6\% das lesões por pressão, a presente pesquisa mostrou resultado discordante apontando uma associação entre lesões por pressão com grau de dano moderado no estado de Minas Gerais e São Paulo ${ }^{18}$.

Ainda que estudos anteriores reportem que a ocorrência de quedas estejam relacionadas a algum tipo de dano na proporção de quase um terço do total desse evento, o presente estudo encontrou associação entre queda e nenhum dano, nos estados do Rio de Janeiro e São Paulo ${ }^{19-20}$.

As falhas durante a assistência à saúde associaram-se ao grau de dano leve em todos estados. Tal relação pode indicar a presença de problemas que poderiam causar danos maiores ao paciente, contudo foram mitigados antes de alcançarem o paciente ou antes de ocasionar dano de grau moderado ou grave ${ }^{21}$.

O óbito, no presente levantamento, esteve relacionado às falhas durante assistência à saúde. Esse achado está em consonância com estudo anterior que reportou as falhas durante assistência como a principal causa dos eventos adversos que resultaram em morte, tendo como principais notificadores Minas Gerais e São Paulo ${ }^{22}$.

Notou-se que os estados do Rio de Janeiro e Espírito Santo tiveram baixo percentual de notificações de incidentes ao longo da série histórica, apresentando tendência decrescente em relação a essas informações. Levando em consideração a população desses estados, a complexidade hospitalar e econômica, pode haver a presença de fatores concatenados à subnotificação como aqueles citados em outras pesquisas que apontaram notificação focada em eventos mais graves, falta de conhecimento sobre 0 tema e centralização da notificação no profissional enfermeiro ${ }^{23-24}$. 
A estacionariedade na notificação de incidentes e eventos adversos evidenciados nos estados de Minas Gerais e São Paulo apontam relação com ações de vigilância constante, ações visíveis e sustentáveis na notificação de incidentes, assim como reportado em outro estudo ${ }^{25}$.

O número de notificações de incidentes por faixa etária apresentou tendência crescente em todos os estados do Sudeste à medida que idade aumenta. Esses achados estão em concordância com outro estudo que também observou aumento na distribuição de incidentes naquelas faixas etárias entre 13 a 59 anos e naqueles com 60 anos ou mais ${ }^{26}$.

\section{CONCLUSÃO}

Portanto, o presente estudo caracterizou as notificações relacionadas a segurança do paciente entre julho de 2019 a junho de 2020 na região sudeste evidenciando um elevado percentual de eventos adversos com predomínio daqueles classificados como leve e moderado. Tal estudo mostrou elevada prevalência das falhas durante a assistência à saúde, daquelas envolvendo cateteres venosos e lesões por pressão. Notou-se ainda, que tanto em Minas Gerais quanto em são Paulo, as lesões por pressão estiveram associadas a eventos adversos de grau moderado, enquanto que no Rio de Janeiro e Espírito Santo as falhas envolvendo cateteres venosos apresentaram relação com eventos adversos de gradação leve. Cabe destacar, quanto a número de notificações de incidentes e eventos adversos relacionados a segurança do paciente ao longo da série temporal, que o Rio de Janeiro e Espírito Santo tiveram tendência decrescente ao longo do tempo pesquisado o que pode representar indícios de subnotificações nessas unidades federativas.

Diante do exposto, destaca-se a importância da implementação de Programas de Capacitação multiprofissional e treinamento com foco na segurança do paciente; implantação de ferramentas gerenciais para diagnóstico de problemas relacionados às falhas e suas causas raízes e o estabelecimento de protocolos administrativos e assistenciais com foco em medidas preventivas, para evitar incidentes, e de atenuação, para reduzir o grau dos danos que recaem sobre o paciente. Por fim, é de extrema relevância a manutenção dos sistemas de vigilância de incidentes e eventos adversos, associados a segurança do paciente, alinhados com o monitoramento e avaliação contínua a fim de evitar a subnotificação de falhas na assistência e gestão nas unidades de saúde.

\section{Conflito de interesses}

Os autores declaram não ter conflito de interesses de origem financeira, política, acadêmica ou comercial com nenhuma instituição. 


\section{REFERÊNCIAS}

1. WHO - World Health Organization. The Final Technical Report for The Conceptual Framework for the International Classification for Patient Safety (v1.1). [Online]; 2009 [citado em 2021 Jul 2919]. Disponível em: http://www.who.int/patientsafety/implementation/taxonomy/icps_technical_report_en.pdf

2. Institute of Medicine (US) Committee on Quality of Health Care in America, Kohn LT, Corrigan JM, Donaldson MS, eds. To Err is Human: Building a Safer Health System. Washington (DC): National Academies Press (US); 2000. doi: https://doi.org/10.17226/9728

3. Mendes GHS, Mirandola TBS. Acreditação hospitalar como estratégia de melhoria: impacto em seis hospitais acreditados. Gest Prod. 2015;22(3):636-48. doi: https://doi.org/10.1590/0104-530X1226-14

4. World Health Organization (WHO). World Alliance for Patient Safety. Forward Programme 2008-2009. Geneva (Switzerland): World Health Organization; 2008. [citado em 2021 Jul 2919]. Disponível em: https://apps.who.int/iris/bitstream/handle/10665/70460/WHO_IER_PSP_2008.04_eng.pdf?sequence=1\&isAllowed=y

5. Zanetti ACB, Gabriel CS, Dias BM, Bernardes A, Moura AA, Gabriel AB, et al. Assessment of the incidence and preventability of adverse events in hospitals: an integrative review. Rev Gaucha Enferm 2020;41:e20190364. doi: https://doi.org/10.1590/1983-1447.2020.20190364

6. Aitken M, Gorokhovich L. Advancing the Responsible Use of Medicines: Applying Levers for Change. Parsippany (NJ): IMS Institute for Healthcare Informatics; 2012. [citado em 2021 Jul 18]. Disponível em: https://ssrn.com/abstract=2222541

7. WHO guidelines for safe surgery 2009: safe surgery saves lives. Geneva: World Health Organization; 2009. [citado em 2021 Jul 18]. Disponível em: http://apps.who.int/iris/bitstream/handle/10665/44185/9789241598552_eng.pdf

8. Agência Nacional de Vigilância Sanitária (Brasil). Gestão de Riscos e Investigação de Eventos Adversos Relacionados à Assistência à Saúde. Brasília: Anvisa, 2017. [citado em 2020 Dez 29]. Disponível em: https://www.segurancadopaciente.com.br/wp-content/uploads/2017/09/Caderno-7-Gest\%C3\%A3o-de-Riscos-e-Investiga\%C3\%A7\%C3\%A3o-de-Eventos-Adversos-Relacionados-\%C3\%A0-Assist\%C3\%AAncia-\%C3\%A0-Sa\%C3\%BAde.pdf

9. Chamberlain-Webber J. Seven steps to patient safety. Professional nurse. 2004;20(3):10-4. doi: http:// dx.doi.org/10.1016/S1134-282X(08)74725-0

10. Agência Nacional de Vigilância Sanitária (Brasil). Incidentes relacionados à assistência à saúde - Resultados das notificações realizadas no Notivisa - Brasil, julho de 2019 a junho de 2020. Brasília: Anvisa, 2020. [citado em 2020 Dez 29]. Disponível em: https://www.gov.br/anvisa/pt-br/centraisdeconteudo/publicacoes/servicosdesaude/relatorios-de-notificacao-dos-estados/eventos-adversos

11. Agência Nacional de Vigilância Sanitária (Brasil). Implantação do Núcleo de Segurança do Paciente em Serviços de Saúde. Brasília: Anvisa, 2015 [citado em 2020 Dez 29]. Disponível em: https://www.saude. go.gov.br/images/imagens_migradas/upload/arquivos/2017-09/2016-anvisa---caderno-6---implantacao-nucleo-de-seguranca.pdf

12. Sinclair D, Croskerry P. Patient safety and diagnostic error: tips for your next shift. Can Fam Physician. 2010;56(1):28-30. Disponível em: https://www.ncbi.nlm.nih.gov/pubmed/20090077

13. Suliman M, Saleh W, Al-Shiekh H, Taan W, AlBashtawy M. The Incidence of Peripheral Intravenous Catheter Phlebitis and Risk Factors among Pediatric Patients. J Pediatr Nurs. 2020; 50:89-93 doi: https:// doi.org/10.1016/j.pedn.2019.11.006

14. Yoshida T, Silva A, Simoes LLP, Guimaraes RA. Incidence of Central Venous Catheter-Related Bloodstream Infections: Evaluation of Bundle Prevention in Two Intensive Care Units in Central Brazil. T ScientificWorldJournal. 2019;2019:1025032. doi: https://doi.org/10.1155/2019/1025032

15. Bereded DT, Salih MH, Abebe AE. Prevalence and risk factors of pressure ulcer in hospitalized adult patients; a single center study from Ethiopia. BMC Res Notes. 2018;11(1):847. 10.1186/s13104-018-39487. doi: https://doi.org/10.1186/s13104-018-3948-7 
16. Bicer EK, Gucluel Y, Turker M, Kepicoglu NA, Sekerci YG, Say A. Pressure Ulcer Prevalence, Incidence, Risk, Clinical Features, and Outcomes Among Patients in a Turkish Hospital: A Cross-sectional, Retrospective Study. Wound Manag Prev. 2019;65(2):20-8. Disponível em: https://www.hmpgloballearningnetwork.com/site/wmp/article/pressure-ulcer-prevalence-incidence-risk-clinical-features-and-outcomes-among-patients

17. Miliani K, Taravella R, Thillard D, Chauvin V, Martin E, Edouard S, et al. Peripheral Venous Catheter-Related Adverse Events: Evaluation from a Multicentre Epidemiological Study in France (the CATHEVAL Project). PLoS One. 2017;12(1):e0168637. doi: https://doi.org/10.1371/journal.pone.0168637

18. Pacha HHP, Faria JIL, Oliveira KA, Beccaria LM. Pressure Ulcer in Intensive Care Units: a case-control study. Rev Bras Enferm. 2018;71(6):3027-34. doi: https://doi.org/10.1590/0034-7167-2017-0950

19. Bouldin EL, Andresen EM, Dunton NE, Simon M, Waters TM, Liu M, et al. Falls among adult patients hospitalized in the United States: prevalence and trends. J Patient Saf. 2013;9(1):13-7. doi: https://doi. org/10.1097/PTS.0b013e3182699b64

20. Stevens JA, Mack KA, Paulozzi LJ, Ballesteros MF. Self-reported falls and fall-related injuries among persons aged $\geq 65$ years-united states, 2006. Journal of Safety Research 2008;39:345-9. doi: https://doi. org/10.1016/j.jsr.2008.05.002

21. Hughes RG. Tools and Strategies for Quality Improvement and Patient Safety. In: Hughes RG, editor. Patient Safety and Quality: An Evidence-Based Handbook for Nurses. Advances in Patient Safety. Rockville (MD)2008. Disponível em: https://www.researchgate.net/profile/Ronda-Hughes/publication/49843307 Tools_and_Strategies_for_Quality_Improvement_and_Patient_Safety/links/573094e508ae6cca19a1e9b1/ Tools-and-Strategies-for-Quality-Improvement-and-Patient-Safety.pdf

22. Maia CS, Freitas DRC, Gallo LG, Araújo WN. Notificações de eventos adversos relacionados com a assistência à saúde que levaram a óbitos no Brasil, 2014-2016. Epidemiol. Serv. Saúde [Internet]. 2018 [acesso em 2020 dez 23] ; 27(2): e2017320. doi: https://doi.org/10.5123/s1679-49742018000200004

23. Alves MFT, Carvalho DS, Albuquerque GSC. Motivos para a não notificação de incidentes de segurança do paciente por profissionais de saúde: revisão integrativa. Ciênc. saúde coletiva [Internet]. 2019 Aug [acesso em 2020 Dez 23]; 24(8): 2895-2908. doi: http://dx.doi.org/10.1590/1413-81232018248.23912017

24. Harper ML, Helmreich RL. Identifying Barriers to the Success of a Reporting System. In: Henriksen K, Battles JB, Marks ES, Lewin DI, editors. Advances in Patient Safety: From Research to Implementation (Volume 3: Implementation Issues). Advances in Patient Safety. Rockville (MD)2005. Disponível em: http:// www.ahrq.gov/professionals/quality-patient-safety/patient-safety-resources/resources/advances-in-patient-safety/vol3/Harper.pdf

25. Mitchell I, Schuster A, Smith K, Pronovost $P$, Wu A. Patient safety incident reporting: a qualitative study of thoughts and perceptions of experts 15 years after 'To Err is Human'. BMJ Qual Saf. 2016;25(2):92-9. doi: https://doi.org/10.1136/bmjqs-2015-004405

26. Lorenzini E, Santi JA, Bao AC. [Patient safety: analysis of the incidents notified in a hospital, in south of Brazil]. Rev Gaucha Enferm. 2014;35(2):121-7. doi: https://doi.org/10.1590/1983-1447.2014.02.44370

RECEBIDO: 05/12/2020

ACEITO: 15/10/2021 